\section{$\underset{\substack{\text { hommes } \\ \text { \& migrations }}}{ }$}

\section{Hommes \& migrations}

Revue française de référence sur les dynamiques

migratoires

\section{$1285 \mid 2010$}

L'appel du pied

\title{
Yeso Amalfi (1950-51)
}

Une vedette brésilienne à l'OGC Nice

\section{Yvan Gastaut}

\section{(2) OpenEdition}

1 Journals

\section{Édition électronique}

URL : http://journals.openedition.org/hommesmigrations/1174

DOI : 10.4000/hommesmigrations. 1174

ISSN : 2262-3353

Éditeur

Musée national de l'histoire de l'immigration

\section{Édition imprimée}

Date de publication : 1 mai 2010

Pagination : $32-47$

ISSN : 1142-852X

\section{Référence électronique}

Yvan Gastaut, "Yeso Amalfi (1950-51) », Hommes \& migrations [En ligne], 1285 | 2010, mis en ligne le 29 mai 2013, consulté le 01 mai 2019. URL : http://journals.openedition.org/hommesmigrations/1174 ; DOI : 10.4000/hommesmigrations. 1174 


\section{Yeso Amalfi (1950-51) Une vedette brésilienne à l'OGC Nice}

Par Yvan Gastaut, maître de conférences, université de Nice

Au début des années cinquante, l'OGC Nice embauche un joueur originaire du Brésil, Yeso Amalfi, qui va profondément marquer l'histoire du club.

Talentueux et fantasque, cet attaquant enflamme les foules et cristallise les passions. Alliant le génie du jeu et un sens certain de la mise en scène, Amalfi est adulé par les médias à l'ỉmage des "stars" contemporaines. "Le beau Yeso" n"est pourtant resté à Nice qu'une seule saison. Le temps de construire son image, d'asseoir sa valeur sportive et de négocier son transfert. Le temps pour la Côte d'Azur de vibrer à l'heure brésilienne. 
Le football est une composante importante de la vie sportive et culturelle azuréenne depuis les années vingt. Amateurs ou professionnels ont foulé les pelouses de la région avec un engouement partagé $e^{(1)}$. Le ballon rond est aussi une source de passion pour l'opinion azuréenne et la commémoration des cent ans de l'OGC Nice, ponctuée par une remarquable exposition en 2004-2005(2) ainsi que par un ouvrage de Michel Oreggia, président du club des supporters saluant également le centenaire $^{(3)}$, a prouvé combien ce club a marqué l'identité locale. Des moments forts, des figures ont jalonné l'histoire du "gym", devenu professionnel dès la mise en place d'un premier championnat de ce type en 1932.

La plus belle séquence du club se situe incontestablement dans les années cinquante : Nice qui jusqu'alors n'avait jamais été sacré Champion de France de première division a remporté à quatre reprises le titre $(1950-51 ; 1951-52$; 1955-56 ; 195859), à deux reprises la Coupe de France $(1951-52 ; 1953-54)$ et a disputé deux quarts de finale de la Coupe des clubs champions (1956-57; 1959-60).

Faire de l'OGC Nice l'un des meilleurs clubs de la France d'après-guerre relève d'une ambition politique. À la fin des années quarante, la municipalité, sous l'impulsion de Jean Médecin, décide de tout mettre en ceuvre pour que la ville possède une grande équipe. Elle n'hésite donc pas à financer abondamment le club qui fonctionne alors autour d'un comité tripartite de gestion rassemblant la municipalité, le Comité des fêtes et les représentants effectifs du "gym" dirigé par un triumvirat composé par le président François Sattegna, l'avocat Jacques Cotta et Jean Médecin lui-même. Lors de la saison 1947-48, un gros effort de recrutement porte immédiatement ses fruits : l'OGC Nice devient champion de France de deuxième division (37 victoires, 10 nuls, 9 défaites) et accède à l'élite. Jean Médecin prolonge les efforts de la municipalité : le club finit à la $7^{\mathrm{e}}$ place avec des hauts et des bas lors de la saison 1948-49 et à la $5^{\mathrm{e}}$ place lors de la saison 1949-50.

Avec le professionnalisme, le football a provoqué depuis les années trente un intensif déplacement de joueurs suscitant un cosmopolitisme original, peu commun à la société française de la période d'après-guerre. Les nations les plus diverses sont représentées dans les équipes dans la limite d'une réglementation qui évolue sans cesse. Club d'élite, l'OGC Nice, sans jamais oublier le vivier local, a cherché à enrôler les plus brillants joueurs étrangers disponibles sur le marché, capables à la fois d'apporter un atout supplémentaire dans le jeu et d'attiser la curiosité des supporters. Chaque nouvel arrivant offre l'occasion de se plonger, non sans stéréotypes, dans une autre culture du football, technique et tactique et par ce biais dans la culture de l'Autre.

Plusieurs joueurs étrangers ont marqué de leur empreinte l'équipe niçoise du premier titre en 1950-51, d'autant que le règlement autorisait désormais chaque 
club à aligner non plus deux mais trois étrangers sur la feuille de match. Si on ne peut prendre en compte les quatre joueurs "indigènes" ou "musulmans", Abdelaziz Ben Tifour ${ }^{(4)}$, Moktar Ben Nacef ${ }^{(5)}$, Hassan M'Jid ${ }^{(6)}$ et Mohammed Firoud ${ }^{(7)}$ qui font encore partie de l'Empire français, il faut compter trois Suédois, Per Uno

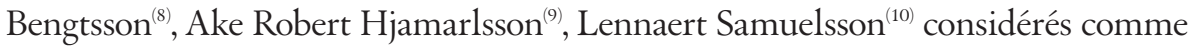
efficaces, rugueux et combatifs, et surtout un Brésilien, Yeso Amalfi, véritable vedette du monde du football, qui défraye la chronique par son comportement imprévisible et par ses frasques en dehors des terrains.

Grâce au football, Nice vit à l'heure brésilienne pendant une saison et, à l'instar de leur intérêt constant dans l'histoire pour cette lointaine terre sud-américaine ${ }^{(11)}$, les Niçois profitent de la présence de Yeso Amalfi pour exprimer plus qu'un attachement, une véritable passion. Il faut dire que les contours du personnage vont largement contribuer à cet enthousiasme pour un sport, le football, un pays, le Brésil et une vedette facétieuse, Amalfi.

\section{Une arrivée remarquée, des débuts difficiles}

Yeso Amalfi, né en 1925 à Sao Paulo, est le fils d'une Italienne et de l'un des plus importants pharmaciens de la ville qui possède quatre drugstores et un laboratoire. Chez les Amalfi, on est pharmacien de père en fils : Yeso, doué à l'école, a presque fini ses études en 1949, il ne lui reste qu'une année de faculté pour qu'il obtienne son diplôme et puisse ainsi succéder à son père. Mais il est aussi bon athlète: adolescent, il pratique la boxe et devient champion du Brésil amateur des poids moyens. Lors d'un combat officiel, il étale son adversaire au second round : sa mère, effarée par la violence de ce sport et inquiète pour son avenir lui fait jurer de raccrocher les gants. Il se tourne alors vers le football où il excelle également.

Il devient champion du Brésil junior puis trois fois champion du Brésil en 1945, 46 et 47 avec le FC Sao Paulo où il joue avec le célèbre avant-centre Léonidas. À cette époque, il porte à 15 reprises le maillot de la prestigieuse équipe du Brésil dont il est la coqueluche. Les gens viennent au stade spécialement pour le voir, certains tirent en l'air des coups de feu en scandant son nom, signe d'enthousiasme. Lorsqu'il quitte le stade, c'est du délire: un jour la foule s'en empare, des centaines de gens l'embrassent au risque de l'étouffer. Transféré en Argentine à Boca Junior de Buenos Aires, il devient l'idole du public et se lie d'amitié avec le président Juan Domingo Peron et sa femme Evita avec laquelle il entretient une relation de séduction, lui offrant souvent des fleurs lorsqu'elle se rend au stade. Puis il émigre en Uruguay au 
Penarol de Montevideo où il côtoie la plupart des internationaux qui remportent la Coupe du monde de 1950 au Brésil : il connaît un premier exil et s'éloigne de la sélection nationale. D'autant que sa saison 1949-50 est assez terne dans la mesure où son père le réclame dans sa boutique de Sao Paulo: Yeso se plie à l'autorité paternelle et ne joue plus au football que par intermittences au Palmeiras de Sao Paulo, parlant même d'arrêter sa carrière. Mais il se marie et veut voyager, rêvant de la France, de Paris, de la Côte d'Azur où il aimerait s'installer pour quelque temps. Trois hommes principalement ont réglé le transfert d'Amalfi à Nice : le dirigeant niçois, Mario Albert, chaud partisan de recruter une vedette brésilienne, l'exentraîneur antibois, Valère de Besvocony, fixé en Amérique du Sud dans les milieux du football et doté d'un solide réseau de relations, et surtout l'agent arménien d'Amalfi, Arthur Bogossian, bottier de luxe à Paris dans le quartier de Passy et spécialisé dans le placement des joueurs sud-américains.

Le 2 août 1950, Yeso Amalfi arrive en rade de Cannes à bord du paquebot Conte Grande Biancamano en provenance de Rio. Immédiatement, journalistes sportifs comme Émile Laurence de Nice-Matin ou Jean Allègre de L'Espoir, supporters et curieux accueillent ce bel athlète aux cheveux noirs ondulés arborant une moustache en "coup de pinceau" qui lui donne des allures de Mexicain et l'accompagnent en direction d'un restaurant cannois Le Petit Brouant rue Deloye où il est attendu par les dirigeants niçois ${ }^{(12)}$. Son premier souci : la condition physique qu'il craint avoir perdue après quinze jours de traversée.

Installé dans un hôtel sans confort près de la gare, il est convié dès le lendemain au stade du Ray pour y subir des tests physiques et techniques : les dirigeants veulent s'assurer de la qualité de la "marchandise" avant de le faire signer. Le Brésilien s'en trouve humilié comme il l'avouera par la suite ${ }^{(13)}$, mais les tests sont concluants : sa démonstration technique impromptue, et notamment la qualité de ses jongles, impressionne les journalistes présents à l'image de Tony Bessy de Nice-Matin et correspondant de France Football ${ }^{14)}$. Il signe dans la foulée son contrat professionnel au siège de l'OGC Nice sous le regard de l'adjoint aux sports de la municipalité Auguste Vérola.

Quelques jours plus tard, le 12 août au stade de St-Augustin, Yeso Amalfi est la principale attraction d'une rencontre de pré-saison entre les professionnels et les amateurs du "gym". On le décrit comme une "authentique vedette (15)" mais aussi comme un "fantaisiste" doutant de ses capacités d'adaptation. Saura-t-il se plier aux exigences de la rigoureuse mais efficace méthode du "WM" telle qu'on la pratique en France et qui laisse peu de place aux artistes ? Quoi qu'il en soit, le public ne regrette pas d'être venu voir ce phénomène qui livre un véritable récital de football (les professionnels l'emportent 5 buts à 0 ), très élégant sur le terrain, paraissant jouer 
avec une "déconcertante facilité" et parfois une "incroyable aisance". C'est selon les propos des quotidiens niçois à l'unisson une "excellente opération" pour le club" Pourtant, Amalfi connaît de rapides problèmes d'adaptation, souffrant du mal du pays, à tel point qu'il parle de quitter Nice dès le début septembre, avant même d'avoir pu jouer en match officiel ${ }^{(17)}$.

En moins de deux mois, le joueur est parvenu à diviser dirigeants et supporters niçois en deux clans, entre admirateurs et détracteurs. Ceux qui pensent que le succès de l'OGC Nice passe par l'effort et le travail rejettent ce "danseur inefficace" et fustigent sa dédaigneuse désinvolture, tandis que ceux qui sont sensibles au beau jeu et aux artistes le défendent contre cet "ostracisme tenace".

Elly Rous, l'entraîneur des Aiglons, fait partie de la première catégorie et n'aime pas Amalfi. Il refuse de l'aligner lors des premières journées de championnat en septembre, estimant tantôt que sa licence n'était pas encore homologuée ou qu'il n'était pas encore en forme : en réalité, le Brésilien est mis sur la touche parce qu'il refuse de se plier à la discipline imposée.

\section{Un phare à éclipses}

Après maintes polémiques, la vedette brésilienne fait ses débuts lors de la quatrième rencontre à Sochaux, perdue (2-3) début octobre 1950. Le commentaire de Fernand Albaret dans France Football est mitigé : certes la classe, la félinité, l'autorité dans la conduite de balle, la précision dans les passes ; mais aucun effort, un joueur immobile, "victime de la mouche tsé tsé", qui a fini le match "avec sa chemisette intacte", ce qui aggrave encore les problèmes d'une attaque niçoise peu performante. L'entraîneur sochalien Paul Wartel livre son analyse : "Amalfi est certainement un joueur de classe. Encore faut-il qu'il s'assimile à un football qui va encore beaucoup trop vite pour lui". En comparaison, le Norvégien de Sochaux Paulsen se situe à l'opposé : laborieux, accroché au ballon, épuisé par ses courses. Elly Rous déclare, désabusé : "Je vous échange Paulsen contre mon phénomène Amalfi $i^{(18)}$." L'entraîneur, préoccupé par le mauvais classement de l'équipe, supporte mal les caprices de sa vedette.

Pourtant, fin novembre, la belle victoire sur Strasbourg 4 buts à 1 révèle enfin le talent du Brésilien qui, devenu remplaçant et ne s'entraînant qu'avec les amateurs, profite de la blessure de Bengtsson pour retrouver l'équipe professionnelle et produire une partie exceptionnelle. Il régale les spectateurs en faisant marquer trois buts et en signant lui-même le quatrième sur une reprise de volée fulgurante. À la fin du match, le préfet des Alpes-Maritimes, Georges Hutin, ancien footballeur et boxeur toujours passionné de sport et le député-maire Jean Médecin sont tellement 
éblouis qu'ils viennent saluer la vedette sud-américaine dans les vestiaires ${ }^{(19)}$. Les "amalfistes" de la première heure prennent alors sa défense en raillant les "néoamalfistes" qui se révèlent "aussi nombreux que les FFI au soir de la Libération". NiceMatin milite pour la reconnaissance définitive du talent d'Amalfi : "Il faut que l'on s'adapte à ce grand footballeur et pas le contraire ${ }^{201}$."

Mais l'ambiance reste mauvaise et faute d'avoir pu redresser la barre, Elly Rous en conflit avec Amalfi et depuis quelque temps avec la direction du club est écarté. Et en janvier 1951, après quelques semaines de tentative d'une direction collective, Numa Andoire, ancien joueur de l'équipe de France, Antibois revenu d'Afrique du 
Nord est nommé entraîneur, assisté du directeur sportif Jean Lardi. Les tensions s'apaisent : Andoire comprend beaucoup mieux Amalfi avec lequel il noue une réelle complicité. Il décide de planifier les entraînements : les défenseurs viennent à $9 \mathrm{~h}$, les milieux de terrain à $10 \mathrm{~h}$ et les attaquants à $11 \mathrm{~h}$ pour des exercices spécifiques. Cette stratégie porte ses fruits : Amalfi et Bergtsson sont définitivement associés en attaque avec le buteur Courteaux qui marquera à 27 reprises au cours de la saison 1950-51 et les Aiglons enchaînent désormais les bons résultats ${ }^{(21)}$.

Les progrès de l'OGC Nice ne mettent toutefois pas un terme aux frasques de son Brésilien qui n'exprime son talent que par intermittences. En janvier 1951, considérant que le match à Montpellier n'est pas important, il a failli ne pas prendre le train ${ }^{(22)}$. Quelques jours plus tard, lors d'un match de préparation sur la pelouse boueuse du stade de Saint-Maurice, il réussit à ne pas se salir, évitant tout contact avec l'adversaire. À la fin du mois,

Au total, la vedette brésilienne, si décisive pour l'équipe selon les observateurs, n'a joué en championnat que 17 matchs sur 38, ne marquant que 6 buts : son rayonnement est aussi décisif sur le terrain qu'en dehors. Yeso Amalfi obtient des dirigeants niçois qu'ils lui octroient quinze jours de repos pour "lassitude générale".

Lors du match décisif pour la course au titre contre Lille, Amalfi enflamme le match et les Aiglons l'emportent brillamment 4 à 1. "Pour la première fois dans l'histoire du football européen" selon Paris Match $^{(23)}$, les 20000 spectateurs du stade du Ray ont fait une ovation "enthousiaste et délirante", debout les bras au ciel pendant dix minutes à leur idole lorsqu'il marque le troisième but, un "chef-d'ceuvre sensationnel". Amalfi l'obtient à la suite d'une passe d'Antoine Bonifaci et après avoir esquivé à l'aide de ses seules feintes de corps cinq adversaires. Une fois le ballon dans les filets, le Brésilien fait un saut en l'air tout à fait excentrique qu'il répète à trois reprises puis renouant avec la tradition des athlètes antiques vainqueurs d'un marathon ou d'une épreuve de javelot, s'agenouille et prostré au sol, baise le gazon. À quelques minutes de la fin, exaspéré par le talent du Brésilien, le défenseur néerlandais de Lille Van Den Hart blesse intentionnellement la vedette niçoise d'un violent coup de pied au mollet gauche : la foule menace d'envahir le stade pour s'en prendre au bourreau.

Dernière surprise avant le titre de champion de France lors de la dernière journée le 27 mai 1951, l'OGCN doit jouer son match décisif à Paris contre le Stade Français, lanterne rouge du championnat et condamné à la descente en deuxième division. La victoire est indispensable et le club mobilise ses énergies. Or, contre toute attente Amalfi demande au Comité de gestion, sous couvert de son entraîneur, de ne pas jouer car les terrains parisiens et surtout le "maudit" terrain de Colombes lui sont 
défavorables et il ne veut pas porter la responsabilité d'une médiocre prestation. À l'annonce de la victoire des Aiglons 4 buts à 0 , synonyme de sacre de champion de France à la différence de but devant Lille ${ }^{(24)}$, les supporters niçois défilent spontanément en ville à la recherche d'Amalfi que l'on retrouve facilement puisqu'il se trouve dans les bureaux de Nice-Matin qui a mis en place un système de relais technique avec Colombes permettant de suivre l'évolution du score en direct ${ }^{(25)}$. Sans avoir pris part au match décisif, la vedette est portée en triomphe dans toute la ville : absent sur le terrain, il apparait pourtant comme le grand triomphateur du jour. France Football ne peut s'empêcher d'ironiser sur la situation: "Nous comprenons mieux qu'il n'ait pas jugé utile de se déplacer ${ }^{(26) . " ~ L e ~ r e t o u r ~ d e s ~ h e ́ r o s ~ l e ~} 4$ juin 1951 se fait dans une ambiance de liesse ${ }^{(27)}$ : un cortège rassemblant les joueurs accompagnés par une foule dense de plusieurs milliers de personnes parade en ville, sur la promenade des Anglais aux abords du siège du Comité des fêtes ${ }^{(28)}$. Avec le capitaine Désir Carré et l'enfant de Villefranche, André Bonifaci, Amalfi, parmi les plus acclamés, n'hésite pas à mettre en scène sa popularité. Reçu avec son équipe au cours d'une réception organisée par le maire Jean Médecin à la villa Masséna, le 6 juin, le Brésilien reçoit une récompense pour son talent. Sous l'égide du club des supporters, une fête populaire est organisée au bar Le Rallye rue Pertinax : Amalfi s'y rend de bonne grâce pour se mêler à ses admirateurs.

Au total, la vedette brésilienne, si décisive pour l'équipe selon les observateurs, n'a joué en championnat que 17 matchs sur 38, ne marquant que 6 buts: son rayonnement est aussi décisif sur le terrain qu'en dehors. Cette inconstance a incité un journaliste parisien à le nommer "monsieur 20 minutes" pour signifier son temps d'investissement dans une rencontre.

\section{L'OGC Nice avec son Brésilien à la Copa Rio : une consécration}

Auréolé de son titre de champion de France, l'OGC Nice a la bonne surprise d'être invité à la "Coupe des champions" de Rio de Janeiro ${ }^{(29)}$ au début du mois de juillet 1951 à la suite de la défection de Barcelone. Ce tournoi lancé par Jules Rimet comme complément à la Coupe du monde des nations, rassemblant huit des meilleurs clubs de la planète, préfigure d'une certaine manière l'émergence des compétitions internationales et intercontinentales entre les clubs, comme la Coupe des clubs champions sur le continent européen quelques années plus tard.

Honorés, les Niçois acceptent de relever le challenge et prennent les choses au sérieux. Deux Aiglons sont particulièrement heureux : Numa Andoire qui retrouve 
vingt ans après le Brésil où il avait joué un match amical avec l'équipe de France avant la Coupe du monde uruguayenne de $1930^{(30)}$ et surtout Yeso Amalfi auquel on accorde la faveur exceptionnelle d'être accompagné de son épouse, qui retrouve son pays. Pour se préparer au mieux, c'est à La Bollène Vésubie que les Niçois font un stage de "mise au vert". Seul Amalfi manque à l'appel, préférant rester sur le littoral et s'entraîner tous les matins au stade Saint-Maurice avec deux Argentins récemment recrutés, César (dit Pancho) Gonzalès et Luis Carniglia, qui participeront au tournoi de Rio. On apprend que l'OGC Nice rencontrera le Palmeiras de Sao Paulo, ancien club d'Amalfi et l'une des meilleures équipes brésiliennes, la Juventus de Turin et l'Étoile Rouge de Belgrade.

Ce tournoi est pour tous les Niçois l'opportunité de faire un long voyage aérien, ce qui relève encore de l'aventure et fait rêver le public. L'épopée est narrée dans la presse niçoise et parisienne dans les moindres détails. Découvrir le "pays du football" est un émerveillement : le capitaine niçois Désir Carré est sollicité par France Football pour livrer aux lecteurs ses impressions dans un feuilleton en quatre épisodes intitulé "Les quinze jours d'un Niçois au Brésil". Dans le premier, Désir Carré raconte dans les moindres détails la manière dont se passent les 36 heures du voyage aérien Nice-Rio : la cérémonie officielle organisée au départ de l'aéroport de la Californie duquel s'envole le "constellation" affrété par la Pan American World Airways ; le comportement d'un Amalfi tout de blanc vêtu, très prolixe dans l'avion, paradant et monopolisant l'attention sur sa personne et son pays depuis qu'il sait qu'il portera le brassard de capitaine pour la circonstance. La vedette des Aiglons juge impossible qu'une équipe européenne puisse l'emporter dans la Copa Rio ${ }^{(31)}$, mais cela ne l'empêche pas d'échafauder un plan pour battre son ancienne équipe : Pancho Gonzales doit absolument charger la plaque tournante de cette équipe, l'attaquant Jair, associé à Rodrigues, qu'il ne faut pas laisser filer...

Le match contre Palmeiras à Sao Paulo, futur vainqueur de l'épreuve, est entouré de fièvre : les 50000 supporters brésiliens font une ovation à Amalfi qui reçoit tous les honneurs de son ancien $\mathrm{club}^{(32)}$. Dans cette ambiance au parfum particulier, les Aiglons font une excellente première mi-temps, faisant jeu égal avec leurs adversaires sous la houlette de leur vedette brésilienne. Mais, au repos, Amalfi, pour des raisons inexpliquées, est remplacé par Courteaux et Nice s'incline trois buts à zéro. Également battus dans les dernières minutes par la Juventus qui accèdera à la finale (2-3), les Aiglons sauvent l'honneur en disposant de l'Étoile Rouge de Belgrade (2-1) sans pouvoir se qualifier pour les demi-finales.

Selon de nombreux observateurs et le maire, Jean Médecin, au-delà du club, c'est la ville de Nice dans son ensemble qui a beaucoup gagné avec ce voyage : mieux connue 
et appréciée, possible lieu de destination touristique voire d'ancrage professionnel pour les footballeurs à l'image de Yeso Amalfi. La presse brésilienne est unanime, l'OGC Nice a porté haut les couleurs de la Côte d'Azur et de la France.

\section{Fantaisiste et facétieux, une vedette hors du commun}

Tout au long de la saison 1950-52, les comportements du Brésilien en dehors du terrain défrayent autant la chronique que son imprévisibilité sur le terrain. À tel point que l'hebdomadaire Paris-Match lui consacre un article en mai 1951 le présentant comme un "héros moderne et romantique" en titrant "La saison 1951 a révélé aux Français un dieu du stade : Amalfi $i^{(33) " .}$

Sa vie privée est exposée. Depuis 1946, il est lié à l'une de ses admiratrices, Sully Costa qu'il rencontre à la fin d'un match au Brésil. Ils se plaisent, vont au dancing et tombent amoureux. Sully Costa devient Miss Brésil fin 1947 sur la plage de Copacabana et suit son compagnon en Argentine et en Uruguay où ils se marient. À Nice, avec son épouse, Amalfi vit rue Amiral de Grasse dans un appartement cossu. Son quartier général est situé au cceur de la ville à la brasserie Le Madrid près de la place Masséna. Malgré sa vie de couple, celui que l'on surnomme "le beau Yeso" reste un séducteur au physique d'hidalgo à la Ramon Novarro et n'hésite pas à s'afficher en public avec d'autres femmes, actrices comme Madeleine Lebaut, danseuses ou mannequins. D'autant qu'il est un danseur admirable : à Copacabana, il a eu pour partenaire Lana Turner avec laquelle des liens d'amitié se sont noués ; à Nice, il danse avec la "bombe argentine" Tilda Thanar.

Aimant les sorties nocturnes, il fréquente les différents cabarets jouant de la samba brésilienne ainsi que les boîtes de nuit de Juan-les-Pins. Un soir, dans un night club de la promenade des Anglais il s'installe tout de blanc vêtu au beau milieu de la pièce et commande du champagne. Face au refus du maître d'hôtel, il menace "Je suis Yeso Amalfi, si on ne me sert pas, je ne jouerai pas dimanche". Le Brésilien est l'une des personnalités les plus en vue du réveillon de la Saint Sylvestre 1950 ${ }^{(34)}$.

Avant l'entraînement, fervent catholique, il se rend à l'église avec un bouquet de fleurs qu'il apporte à la Sainte Vierge. Le dimanche, avant chaque match, il assiste à la messe et prie longuement. Puis parfois, après une rencontre, il lui arrive de sauter dans le train et de filer en Italie pour visiter des églises. Par superstition, il joue toujours avec la même culotte usagée depuis plusieurs années, maintes fois reprisée et sa mascotte est un briquet en or que lui ont offert deux frères boulangers-pâtissiers du quartier Masséna, les frères Pellero, ses supporters et amis de la première heure. 
Coquet, Amalfi est particulièrement sensible à sa coiffure, fréquentant plusieurs salons niçois. Lors des déplacements, il tient absolument à se rendre chez un coiffeur avant le match. Il lui arrive de jouer avec un peigne dans la poche de son short afin de plaquer sa noire chevelure lorsqu'une mèche s'est légèrement déplacée. Lors d'un match amical à St-Raphaël, "le beau Yeso" décide de ne jamais toucher le ballon de la tête pour éviter d'être décoiffé, car les dirigeants locaux souhaitent le photographier à la fin de la partie pour le placer dans leur salle d'honneur.

C'est aussi un bon vivant : toute la semaine il fume des cigarettes qu'il coupe en deux pour limiter la consommation mais s'arrête deux jours avant le match. Amateur de spaghettis, d'escalopes bolognaises et de steaks saignants, il ne refuse jamais une coupe de champagne. Ses manies sont nombreuses : entraînement en chaussures bleu marine à fine semelle de caoutchouc; match en souliers spéciaux si flexibles que l'on peut les plier et les mettre dans une poche. Sa garde-robe, magnifique et excentrique, s'apparente à celle d'un étudiant brésilien fils d'une riche famille: plusieurs dizaines de complets et de gabardines claires parmi lesquels certains sont d'une blancheur immaculée.

Dès son arrivée, il se lie d'amitié avec le tailleur de Picasso, Sapone qui lui donne un conseil dont il tiendra régulièrement compte : "Pour réussir en France, tu dois miser sur ton côté extravagant afin d'attirer l'opinion publique ${ }^{(35)}$." En octobre 1950, pour alimenter le conflit avec son entraîneur Elly Rous, les deux hommes montent un "coup médiatique" qui restera dans les annales du football azuréen : à la fin d'un entraînement, Yeso Amalfi se rend dans le bazar d'un gros commerçant niçois, décroche un matelas et l'installe au beau milieu de la rue. Il s'allonge et fait mine de s'endormir. Les voitures klaxonnent et Yeso hurle: "Voilà ce qu'il résulte d'un entraînement à la française." Sapone ayant convoqué les journalistes, l'affaire remonte jusque dans la presse sportive nationale et internationale. Emmené au poste par la police, il raconte les misères que lui inflige Elly Rous et gagne la confiance du public niçois qui non seulement découvre des gestes de footballeur qu'il ne connaît pas et qui en plus se délecte de ses frasques en dehors du terrain.

\section{Une passion niçoise éphémère mais inoubliable}

À Nice, la popularité de Yeso Amalfi a pris des proportions exceptionnelles d'autant que sa présence dans le club s'est limitée à une seule saison et la moitié des matchs. Dans l'ouvrage Les Cent Ans de l'OGC Nice" Henri Barbaroux de Lamper, supporter inconditionnel du club entre 1947 et 1957, se souvient avant tout d'Amalfi qui a 
laissé dans sa mémoire un souvenir impérissable, tant ses gestes et son jeu étaient spectaculaires et hors du commun ${ }^{(36)}$.

Le "gâteau Amalfi" s'arrache le dimanche dans les pâtisseries de la ville, une nouvelle mode se développe chez les jeunes hommes : se laisser pousser la moustache "à coup de pinceau" en laissant onduler ses cheveux noirs. Le Brésilien est le héros d'une chanson diffusée à partir de décembre 1950 sur les ondes de radio. Un air de samba intitulé "Le Beau Yeso" est joué dans les cabarets niçois par quelques musiciens locaux, inspirés par leurs homologues brésiliens.

Selon les journalistes de Paris Match, "à Nice, il est à la fois le dieu, la vedette, le prophète,

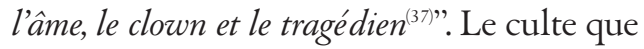
les Niçois lui vouent prend parfois des résonances américaines. Pour ses supporters,

‘J'ai rarement vu un spectacle aussi beau. Entre le ballon et Amalfi, il y a de secrètes correspondances, une sorte de pacte robuste et délicat. C’est un génie... ".' c'est un inspiré du football qui a quelque chose de magique dans son jeu. On s'extasie sur le fait qu'il ne regarde jamais le ballon, mais plutôt l'homme adverse qui le marque. À l'entraînement, il est capable de garder la balle un quart d'heure en l'air sans qu'elle ne tombe dans un exercice intitulé le "jonglage papa".

Le journaliste et ancien joueur Gabriel Hanot le présente comme "le meilleur technicien ayant jamais opéré dans une équipe française". Jean Cocteau, qui assiste rarement à un match de football, est traîné au stade de Saint Maurice par ses amis pour qu'il prenne connaissance du phénomène sud-américain lors d'une rencontre amicale. Le poète est enchanté : "J'ai rarement vu un spectacle aussi beau. Entre le ballon et Amalfi, il y a de secrètes correspondances, une sorte de pacte robuste et délicat. C'est un génie...?' Pablo Picasso présent dans les tribunes pour les matchs des Aiglons est tout aussi admiratif et avoue s'inspirer des figures de style d'Amalfi pour sa propre création.

Le Comité des traditions niçoises le sollicite au moment du carnaval : il apparaît sur le char le plus prestigieux du défilé pour le plus grand bonheur de la population et anime une soirée de gala en février 1951. "Le Beau Yeso" s'essayera même au cinéma en janvier 1953 dans un court-métrage sur son propre personnage, intitulé Monsieur Amalfi dans une réalisation de Gilbert Cocanas sur un scénario de Jean Cornu. Il fréquente à cette occasion les acteurs Micheline Presle et Gino Cervi.

Les supporters sont tellement passionnés qu'un certain nombre d'entre eux créent le "Club des Amalfistes de Nice" dont le slogan est : "Si Yeso quitte Nice, ce ne sera pas vivant". En fin de saison, ce "fan-club" n'a qu'un objectif : faire en sorte qu'Amalfi reste à Nice une saison supplémentaire. Le "Club des Amalfistes" se mobilise notamment pour trouver de l'argent qui permettrait à l'OGC Nice de s'aligner sur 
les propositions financières les plus importantes concernant le joueur brésilien. Signe supplémentaire d'inquiétude, à la fin de la Copa Rio, Amalfi annonce qu'il ne rentre pas à Nice : est-ce provisoire, le temps des vacances? Est-ce définitif ${ }^{38)}$ ? Durant l'été 1951, l'incertitude et la confusion règnent sur l'avenir niçois de la vedette des Aiglons.

Fin juillet des nouvelles rassurantes arrivent de Sao Paulo, Yeso reviendra à Nice au début du mois d'août. Son agent, Arthur Bogossian confirme: "Yeso n'est pas homme d'argent, c'est surtout un sentimental et un émotif : il faut savoir le comprendre et l'aimer ${ }^{(39) "}$. Mais son retour se fait attendre. Yeso Amalfi atterrit finalement le 22 août en fin de soirée à l'aéroport de Nice. De nombreux supporters sont sur place, un défilé de voitures est organisé( ${ }^{(40)}$. Le Brésilien parcourt la promenade des Anglais et l'avenue de la Victoire debout dans une Cadillac, faisant penser selon certains journalistes ironiques à "Jules César rentrant à Rome après la conquête de la Gaule" ou à "Einsenhower défilant dans la Cinquième Avenue après la victoire des Alliés ${ }^{(41)}$ ". Amalfi est surpris et gêné par cet accueil chaleureux qui reste ancré dans sa mémoire comme l'un des plus beaux souvenirs de sa $v^{(42)}$ : des milliers de personnes scandent "Yeso, reste avec nous, reste ici ?', jettent des fleurs sur son passage, agitent drapeaux niçois et brésiliens, brandissent son portrait.

Pendant plus d'une semaine, des discussions serrées s'engagent entre les dirigeants niçois, les émissaires du Torino et l'agent Bogossian. Le 30 août, l'issue est officielle : Yeso sera Turinois la saison suivante. Quelle est la part de décision personnelle du joueur dans la négociation? Difficile de le dire puisque, l'OGC Nice affirme avoir cédé à toutes ses exigences et accuse Amalfi qui a disparu sans prévenir, d'avoir trahi ses engagements et ses supporters ${ }^{(43)}$. Les journalistes, après avoir annoncé le prolongement du contrat, doivent démentir, non sans amertume, à l'image d'un Émile Laurence furieux : "Après avoir manqué dix fois à sa parole, Yeso vient de nous quitter pour se rendre à Turin où il espère obtenir un contrat fastueux. Nous voulons bien y croire et nous le lui souhaitons, mais il laissera dans l'esprit des sportifs niçois le regret d'avoir connu un personnage médiocre, sans parole se dérobant à tous ses engagements. C'est profondément triste car nous espérions beaucoup mieux de lui ${ }^{(4)) " . ~ L e s ~ p r o p o s ~}$ d'Amalfi apportent une autre vision des $\operatorname{choses}^{(45)}$ : sans avoir rien décidé le Brésilien apprend par l'intermédiaire de Bogossian qu'il part pour Turin. Après un retour aussi triomphal, il n'a pas eu le courage d'annoncer son départ autrement que par une lettre adressée au Comité de gestion du club quelques jours plus tard. Il quitte Nice en pleine nuit, le cceur gros.

Au Torino, malgré des prestations en demi-teinte, Amalfi est considéré comme le joueur le plus spectaculaire du championnat italien ${ }^{(46)}$. Mais il s'ennuie, estimant que le championnat français est meilleur et considérant que la vie turinoise est monotone. 
À la fin de la saison 1951-52, il propose ses services à l'OGC Nice qui cette fois-ci refuse sans ménagement. Amer et revanchard, Amalfi décide de signer à l'AS Monaco qui joue en deuxième division pour attirer "ses" supporters niçois. Amalfi ne resta que quelques semaines au pied du rocher, le temps de se frotter à l'OGC Nice lors de rencontres amicales comme ce tournoi sans enjeu à Grasse où, en présence "de nombreuses jolies filles" et de l'OGC Nice, Amalfi livre un récital de football ${ }^{(47)}$.

Dès l'automne 1952, il retrouve la première division en rejoignant le Racing de Paris pour presque trois saisons jusqu'en 1955. En novembre, avec son nouveau club, Amalfi revient à Nice pour une rencontre de championnat un peu particulière. Personne ne l'a oublié : la vedette est acclamée et réitère ses manies d'antan tout en jouant un superbe match à tel point que le Racing l'emporte au stade du Ray (2-1). Yeso avoue avoir voulu faire regretter aux dirigeants niçois leur mépris lorsqu'il a exprimé le désir de revenir à Nice la saison précédente ${ }^{(48)}$.

Pendant la guerre d'Algérie, la moustache rasée pour ne plus être arrêté parce qu'on le prend pour un Arabe, le Red Star en 1955-57 et l'Olympique de Marseille en 1957-59 sont ses deux derniers clubs. Le Brésilien arrête sa carrière en 1959 à 34 ans pour devenir agent de joueur jusqu'en 1964 en France, découvrant notamment Nestor Combin. Nostalgique de sa première année en France passée sur la Côte d'Azur, Yeso revient quelquefois à Nice assister à des matchs, toujours adulé du public laissant toujours espérer un retour possible qui ne viendra jamais. Après avoir quitté la France, il fait le tour du monde puis s'installe dans sa ville natale de Sao Paulo en 1965. En possession de deux propriétés et cinq appartements, il devient grand propriétaire terrien, producteur de café dans l'État de Sao Paulo, puis vit de ses rentes, en demeurant toujours attentif au monde du football.

\section{Conclusion}

L'étude du passage éclair à Nice de Yeso Amalfi, footballeur le plus extraordinaire de son temps, se structure autour de trois axes de réflexions dans le champ de l'histoire culturelle.

Quel statut pour la vedette dans le monde du football ? Jean Eskenazi dans un article de France football, "De l'art d'être vedette" en 1954, évoque Yeso Amalfi comme la vedette par excellence, c'est-à-dire un mal indispensable, un mélange de talent, de popularité et de caprice hollywoodien ${ }^{(49)}$. Comme d'autres figures du football et du monde des arts, le Brésilien a cristallisé une attention passionnée et partagée par un grand nombre de Niçois amateurs de ballon rond. Les médias, attentifs à ses moindres gestes, alimentent une notoriété qui n'est pas nécessairement 
en rapport avec les performances effectives du joueur. Amalfi est un personnage à part dont le sens de l'esthétique sur le terrain est poussé jusqu'à la caricature et dont les frasques dans sa vie privée suscitent admiration et curiosité. L'idolâtrie s'exprime avec une intensité qui a beaucoup de points communs avec notre actualité concernant les "stars" du football. Parmi ses caractéristiques les plus nettes, l'ancrage mémoriel : "Le beau Yeso" est resté dans la légende, dans le souvenir de nombreux supporters, de génération en génération.

Amalfi, Niçois d'adoption, artiste et vedette est aussi une "valeur" monnayable de premier choix. La carrière française d'Amalfi est ainsi motivée par des intérêts financiers incontournables dont l'OGC Nice sera partie prenante. Sa venue à Nice, ses transferts ultérieurs mettent en lumière des pratiques déjà bien installées dans les milieux du football d'après-guerre.

Dans cette belle décennie cinquante de l'OGC Nice, elles seront encore nombreuses les vedettes étrangères venues de pays dotés des meilleures cultures du football pour passionner le public. Mais dans le cour des Niçois, comme beaucoup plus récemment Éric Cantona chez les supporters de Manchester United, Yeso Amalfi conserve une place à part dans le panthéon des joueurs qui ont marqué le club. La superposition de plusieurs images a permis de faire d'Amalfi la figure emblématique du football azuréen d'après-guerre : un talent à l'état pur dans le jeu, une capacité à mettre sa vie privée en scène et surtout une bonne adaptation à la vie niçoise et aux traditions locales.

\section{Notes}

1. Voir le mémoire de maîtrise en histoire de Pierre Magnificat, Le football à Nice dans l'entre-deux-guerres, à travers L'Eclaireur de Nice, sous la direction de Ralph Schor, 2003 et le mémoire de maitrise à l'UFR STAPS de Valère Tricard, Les belles années de l'OGC Nice (1951-52) à travers deux quotidiens locaux : Nice-Matin et L'Espoir, sous la direction d'Yvan Gastaut, 2004.

2. Voir le catalogue de l'exposition présentée à l'Acropolis du 11 décembre 2004 au 30 janvier 2005, "Les 100 ans de l'OGC Nice", Nice, Rom éditions, 2004.

3. Michel Oreggia, OGC Nice, 100 ans de passion, Nice, Giletta Nice-Matin, 2004.

4. Tunisien né à Hammam-lif.

5. Tunisien né à Bizerte.

6. Marocain né à Casablanca.

7. Il s'agit du frère de Kader Firoud le capitaine nîmois, originaire comme lui d'Oran.

8. Attaquant adroit et puissant d'un haut niveau, il arrive en septembre 1950, transféré du Torino. 
9. Cet attaquant solide n'est arrivé à Nice qu'en cours de saison en janvier 1951, prêté par le Torino.

10. Cet arrière n'a joué que 9 matchs de championnat en provenance du Torino avant de rejoindre le club de Göeteborg, mais son passage a été remarqué chez les supporters qui l'ont accompagné avec ferveur à la gare le jour de son départ.

11. Voir le site de la ville de Nice, évoquant "Les Niçois et le Brésil", http://www.nice.fr/mairie_nice_5824.html.

12. Nice-Matin, 29 juillet 1950.

13. Voir Roger Driès, "OGC Nice de toujours", Paris, Solar et Nice, Alpazur éditions 1977 et Roger Driès, Jean Chaussier, "OGC Nice, le roman des Aiglons", Paris, Solar, Alpazur éditions, 1985. Roger Driès évoque dans cet ouvrage les souvenirs de Yeso Amalfi retrouvé par Jean Chaussier, journaliste de Nice Matin en 1976 au Brésil. Chaussier est fébrile à l'idée de le retrouver l'idole de sa jeunesse à Sao Paulo. À 51 ans, marié à une femme de 24 ans et père de deux jeunes enfants, il habite dans un immeuble bourgeois.

14. Nice-Matin, 30 juillet 1950.

15. Nice-Matin, 11 août 1950, article de Tony Bessy et France football, 9 août 1950, article de Fernand Albaret.

16. Nice-Matin, 15 août 1950.

17. Nice-Matin, 9 septembre 1950.

18. France football, 6 octobre 1950.

19. Nice-Matin, article de Mario Brun, 28 novembre 1950.

20. Nice-Matin 13 décembre 1950.

21. Nice-Matin, 28 décembre 1950.

22. Nice-Matin, 14 janvier 1951.

23. Paris Match, 19 mai 1951 , op. cit.

24. Cinq équipes peuvent encore prétendre au titre lors de la dernière journée : Nice, Le Havre, Lille, Reims et Nîmes.

25. Nice Matin, 29 mai 1951. La Ligue nationale de football décide d'interdire toutes les retransmissions radiodiffusées pour conserver l'équité des rencontres.

26. France football, 29 mai 1951.

27. L'Espoir, 5 juin 1951.

28. Voir les images dans le catalogue de l'exposition, "Les 100 ans de l'OGC Nice", op. cit.

29. Ce sera la dernière édition de cette Coupe qui, contestée et coûteuse, disparaît l'année suivante faute de soutiens suffisants. Toutes les équipes présentes dans ce tournoi ne sont pas championnes de leur pays.

30. Numa Andoire a été du voyage lors de la Coupe du monde en Uruguay, mais, cantonné au rôle de remplaçant, il n’a pas joué.

31. France football, 26 juin 1951.

32. Nice-Matin, 29 juin 1951.

33. Paris Match, 19 mai 1951, reportage d'Yves Salgues et Willy Rizzo.

34. Nice-Matin, 3 janvier 1951.

35. Roger Driès, OGC Nice de toujours, op.cit..

36. Les 100 ans de l'OGC Nice, op. cit.

37. Paris Match, 19 mai 1951, op. cit.

38. À la une de Nice-Matin, 8 juillet 1951.

39. Nice-Matin, 7 août 1951.

40. Nice-Matin, 24 août 1951

41. Nice-Matin, 26 aout 1951, article de Mario Brun.

42. Roger Driès, OGC Nice de toujours, op. cit. et Roger Driès, Jean Chaussier, OGC Nice, le roman des Aiglons, op. cit.

43. Nice-Matin, 31 août 1951.

44. Nice-Matin, 1er septembre 1951.

45. Roger Driès, OGC Nice de toujours, op. cit. et Roger Driès, Jean Chaussier, OGC Nice, le roman des Aiglons, op. cit.

46. France football, 10 juillet 1952 .

47. France football, 12 aout 1952.

48. France football, 25 novembre 1952.

49. France football, 5 novembre 1954. 